\title{
Luteolin decreases the UVA-induced autophagy of human skin fibroblasts by scavenging ROS
}

\author{
MIAOMIAO YAN*, ZHONGRONG LIU*, HUILAN YANG, CUIHUA LI, \\ HULIN CHEN, YAN LIU, MINLING ZHAO and YINGJIE ZHU
}

Department of Dermatology, Guangzhou General Hospital of Guangzhou Military Command, Guangzhou, Guangdong 510010, P.R. China

Received May 18, 2015; Accepted April 22, 2016

DOI: $10.3892 / \mathrm{mmr} .2016 .5517$

\begin{abstract}
Luteolin (LUT) is a flavone, which is universally present as a constituent of traditional Chinese herbs, and certain vegetables and spices, and has been demonstrated to exhibit potent radical scavenging and cytoprotective properties. Although LUT has various beneficial effects on health, the effects of LUT on the protection of skin remain to be fully elucidated. The present study investigated whether LUT can protect human skin fibroblasts (HSFs) from ultraviolet (UV) A irradiation. It was found that, following exposure to different doses of UVA irradiation, the HSFs exhibited autophagy, as observed by fluorescence and transmission electron microscopy, and reactive oxygen species (ROS) bursts, analyzed by flow cytometry, to differing degrees. Following incubation with micromolar concentrations of LUT, ROS production decreased and autophagy gradually declined. In addition, the expression of hypoxia-inducible factor- $1 \alpha$ and the classical autophagy-associated proteins, LC3 and Beclin 1 were observed by western blotting. Western blot analysis showed that the expression levels of HIF-1 $\alpha$, LC3-II and Beclin 1 gradually decreased in the UVA-irradiated HSFs following treatment with LUT. These data indicated that UVA-induced autophagy was mediated by ROS, suggesting the possibility of resistance against UV by certain natural antioxidants, including LUT.
\end{abstract}

\section{Introduction}

Flavonoids, which are polyphenolic compounds, have been widely investigated for their antioxidant effects (1).

Correspondence to: Mr. Zhongrong Liu, Department of Dermatology, Guangzhou General Hospital of Guangzhou Military Command, 111 Liuhua Road, Guangzhou, Guangdong 510010, P.R. China

E-mail:pfklzr@163.com

\section{${ }^{*}$ Contributed equally}

Key words: luteolin, ultraviolet, reactive oxygen species, autophagy, human skin fibroblasts
Flavonoids have two classical antioxidant structural components, including a B-ring catechol group, which donates a hydrogen/electron to stabilize a radical species, and a C2-C3 double bond conjugated with an oxo group at $\mathrm{C} 4$, which binds transition metal ions, including iron and copper $(2,3)$. Luteolin (LUT), one of the most common flavonoids found in plants in the form of glycosides, are eventually metabolized by intestinal bacteria, cleaved and glucuronated during uptake in the gut and metabolism in the organism. As LUT and a number of its glycosides fulfill these two structural requirements, it has been suggested that LUT possesses antioxidant properties (4).

A number of signaling events are initiated and driven by oxidative stress. Hydrogen peroxide $\left(\mathrm{H}_{2} \mathrm{O}_{2}\right)$ and other reactive oxygen species (ROS) lead to oxidative stress, and are increased by several stimulants, including ultraviolet (UV) irradiation (5-10), which also decrease levels of anti-oxidant enzymes (11). These features exist in chronologically aged human tissues, particularly skin. These two factors increase ROS production, which leads to alterations in genes, protein structure and function, finally leading the damage of tissues, including the skin. During a process of energy transfer, the superoxide anion, $\mathrm{O}^{2-}$, is produced from endogenous UV-absorbing chromophores (12) into molecular oxygen. The superoxide dismutase (SOD) catalyzes $\mathrm{O}^{2-}$ to produce $\mathrm{H}_{2} \mathrm{O}_{2}$ which can be converted to the reactive hydroxyl radical, $\mathrm{HO} \bullet(8)$. These compounds are ROS, which can activate several downstream proteins.

Human skin is usually exposed to several oxidants, of which UV is the most common, causing ROS burst. Increasing levels of harmful oxygen free radicals are implicated in the pathogenesis of skin carcinoma, the mechanism of skin senescence and other skin diseases (5). The current focus on the bioactivity of the flavonoids is partly due to the potential health benefits of the polyphenolic components present within major dietary constituents. LUT, which is considered to be one of the most important flavonoids, has been reported to resist against several extraneous oxidants (2-4), however, its antioxidant effects against UV remain to be fully elucidated. The present study aimed to investigate the probability of LUT scavenging the ROS induced by UVA irradiation in human skin fibroblasts (HSFs), and to examine the potential mechanism to allow improved skin protection. 


\section{Materials and methods}

Cell culture. HSFs, isolated from the foreskins of children (age, 3-9) following circumcision surgery at the Guangzhou General Hospital of Guangzhou Military Command (Guangzhou, China), were routinely cultured in Dulbecco's modified Eagle's medium (DMEM) supplemented with 10\% newborn calf serum (Gibco; Thermo Fisher Scientific, Inc., Waltham, MA, USA) with $4 \mathrm{mM}$ glutamine, $100 \mathrm{U} / \mathrm{ml}$ penicillin and $100 \mathrm{mg} / \mathrm{ml}$ streptomycin, following homogenization of tissue. The cells were harvested with trypsin when they reached $80 \%$ confluence and were seeded in a 6-well plate at a density of $1 \times 10^{5}$ cells/well at $37^{\circ} \mathrm{C}$. Following incubation for $48 \mathrm{~h}$, the cultured medium was removed. The successfully cultured cells were stored in a nitrogen canister. Cells between passages 4-10 were used in the subsequent experiments. The present study was approved by the ethics committee of the Guangzhou General Hospital of Guangzhou Military Command.

Reagents and antibodies. LUT, glutamine, penicillin, streptomycin, trypsin, formaldehyde, glutaraldehyde, $\mathrm{OsO}_{4}$, uranyl acetate, lead, SDS, doxorubicin (DOX), rhodamine123 [for the detection of mitochondrial membrane potential (MMP)] and dichlorodihydrofluorescein diacetate (DCFH-DA) were purchased from Sigma-Aldrich (St. Louis, MO, USA). 3-(4,5-dimethylthyl-thiazol-2-yl)-2,5-diphenyltetrazolium bromide (MTT) was purchased from Promega Corporation (Madison, WI, USA), and DAPI and radioimmunoprecipitation lysis buffer were purchased from Beyotime Institute of Biotechnology (Haimen, China). LUT and DOX were dissolved in dimethyl sulfoxide (DMSO; Sigma-Aldrich). LUT concentrations of 5, 20 and $40 \mu \mathrm{M}$ were used in the experiments. Unless otherwise specified in the figure legends, values are expressed as concentrations in $\mu \mathrm{M}$. Beclin 1 and LC3 antibodies were obtained from Cell Signaling Technologies, Inc. (Danvers, MA, USA). Primary antibodies against GAPDH, $\beta$-actin and hypoxia-inducible factor (HIF)-1 $\alpha$, and the secondary horseradish-peroxidase-labeled antibodies, were also purchased from Beyotime Institute of Biotechnology.

Irradiation procedure. When the cells reached $80 \%$ confluency, they were irradiated under a Solar UV Simulator (Oriel ${ }^{\circledR}$ Sol-UV-4; Newport Corporation, Irvine, CA, USA). The radiation intensity was measured using a UVX digital radiometer (Ultra-Violet Products, Inc., Uplands, CA, USA) equipped with a UVX-310 sensor. The HSFs were irradiated by $320-400 \mathrm{~nm}$ UVA in single or repetitive $7.2 \mathrm{~J} / \mathrm{cm}^{2}$ low doses. The medium was removed and the cells were washed twice with phosphate-buffered saline (PBS) prior to UV irradiation. The cells were covered with a thin film of PBS during UV exposure, and remained in culture in the maintenance medium following irradiation for $10 \mathrm{~min}$ repeated 3 times. A control group of cells were treated in a similar manner, however, these cells were exposed to normal room lighting. All cells were incubated at $37^{\circ} \mathrm{C}$ and $5 \% \mathrm{CO}_{2}$.

Cell viability assessment. To measure cell viability, the MTT method was used (13). Prior to adding the MTT working solution $(5 \mathrm{mg} / \mathrm{ml})$, the cells were seeded in 96 -well plates at a density of $5 \times 10^{5}$ cells per well overnight, and treated with LUT and UVA, as indicated. Subsequently, the cells were incubated in a $\mathrm{CO}_{2}$ incubator for $4 \mathrm{~h}$. The medium was then replaced with $150 \mu$ DMSO (Sigma-Aldrich) to completely dissolve the formazan crystals. The absorbance of each well was then measured using a plate reader (iMark; Bio-Rad Laboratories, Inc., Hercules, CA, USA) at a test wavelength of $570 \mathrm{~nm}$. Cell viability was calculated using the following equation: Cell viability $=$ absorbance of experiment samples $/$ absorbance of control) x $100 \%$.

Apoptosis assay. Apoptosis was determined using an Annexin V-fluorescein isothiocyanate (FITC)/propidium iodide (PI) staining procedure. In brief, following treatment with UV irradiation and incubation with LUT, the cells were collected and washed twice with ice-cold PBS, followed by incubation with Annexin V-FITC and PI. Fluorescence was measured using a BD FACSCalibur flow cytometer (BD Biosciences, Franklin Lakes, NJ, USA) with an excitation wavelength of $480 \mathrm{~nm}$ through a FL-1 filter $(530 \mathrm{~nm})$ and a FL-2 filter (585 nm).

Cellular ROS measurement. The dichloro-dihydro-fluorescein diacetate (DCFH-DA) fluorescent dye (14) was used for ROS analysis. In brief, following treatment with UVA with or without LUT, the cells were collected and incubated with $10 \mu \mathrm{M} \mathrm{DCFH}-\mathrm{DA}$ at $37^{\circ} \mathrm{C}$ for $30 \mathrm{~min}$. Finally, the cells were washed three times with PBS, and the fluorescence was measured using a flow cytometer through an FL-1 filter with an excitation wavelength of $480 \mathrm{~nm}$.

Analysis of autophagy. Monodansylcadaverine (MDC) has been used as a tracer for autophagic vacuoles previously (15). In the present study, $1 \times 10^{5} / \mathrm{ml}$ cells were seeded on coverslips overnight, and then exposed to UVA with or without LUT, as described above, and rinsed with PBS. The cells were then stained with $50 \mu \mathrm{M} \mathrm{MDC}$ at $37^{\circ} \mathrm{C}$ for $1 \mathrm{~h}$ and examined using a flow cytometer.

Cell morphology assessment. Autophagic vascular organelles (AVOs) were examined by staining the treated cells with MDC (Sigma-Aldrich) (15) for $30 \mathrm{~min}$ at $37^{\circ} \mathrm{C}$. Following washing of the cells with PBS, $4 \%$ formaldehyde was added to fix the cells for $30 \mathrm{~min}$. The cells were observed under a Nikon Intensilight fluorescence microscope (Nikon Corporation, Tokyo, Japan) following washing with PBS three times. To observe nuclear morphology, the cells were incubated with DAPI for $10 \mathrm{~min}$ following fixation with $4 \%$ formaldehyde for $30 \mathrm{~min}$ and washing with PBS. The cells were then observed under a fluorescence microscope following washing with PBS three times.

For transmission electron microscopy, the cells were fixed with $2.5 \%$ glutaraldehyde in $0.1 \mathrm{M}$ phosphate buffer $(\mathrm{pH} 7.4)$, followed by $1 \% \mathrm{OsO}_{4}$. Following dehydration, thin sections $(70 \mathrm{~nm})$ were stained with uranyl acetate and lead for observation under an electron microscope (JSM-6010LA; JEOL, Ltd., Tokyo, Japan) (16).

Western blot analysis. At a density of $1 \times 10^{7}$ cells $/ \mathrm{ml}$, the cells were treated with either $10 \mu 15 \%$ DMSO, LUT alone 

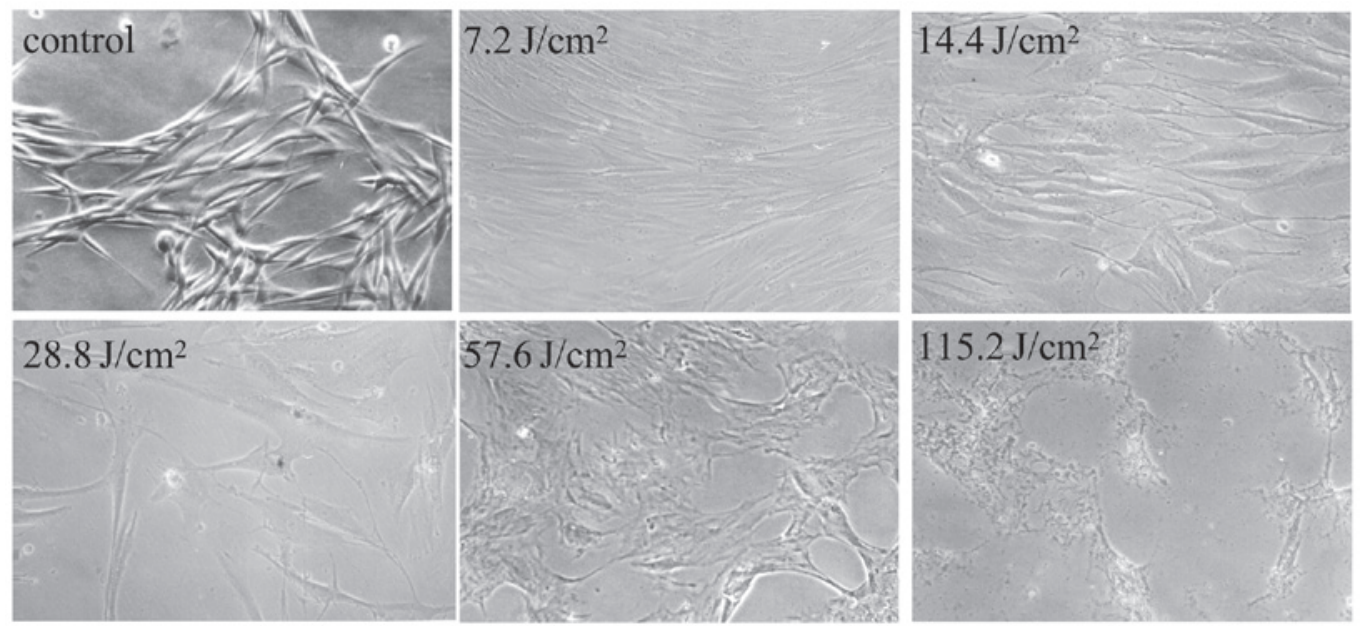

Figure 1. Morphological changes of HSFs under repetitive doses of UVA. HSFs at the forth passage were cultured in a 6 -well plate until $80 \%$ confluent, and then irradiated with repetitive doses of UVA. Following UVA irradiation, HSFs and dose-dependent cell death were observed under a light microscope (magnification, x20). HSFs, human skin fibroblasts; UVA, ultraviolet A.

$(0,5,20$ and $40 \mu \mathrm{M}), 10 \mathrm{ml}$ DOX alone, or with a combination of LUT and DOX at $37^{\circ} \mathrm{C}$ for $48 \mathrm{~h}$, and were harvested at indicated time points. Following a lysis procedure, the lysates were centrifuged at $12,000 \mathrm{~g}$ for $15 \mathrm{~min}$ at $4^{\circ} \mathrm{C}$. Bicinchoninic acid protein assay reagent (Beyotime Institute of Biotechnology) was used to quantify the protein concentrations of the supernatants. The protein $(50 \mathrm{mg})$ from each sample were separated by $30 \%$ SDS-PAGE and transferred to a polyvinylidene fluoride membrane. The membrane was blocked with $5 \%$ non-fat milk and incubated with primary antibodies (dilution, 1:1,000) at room temperature for $2 \mathrm{~h}$, then washed 3 times for 5 min prior to incubation with secondary antibodies at room temperature for $1 \mathrm{~h}$. The bands were detected using a ChemiDoc Touch imaging system (Bio-Rad Laboratories, Inc.).

Statistical analysis. The data are expressed as the mean \pm standard deviation and were analyzed using Student's $t$-test (two-tailed) and SPSS 22.0 (IBM SPSS, Armonk, NY, USA). $\mathrm{P}<0.05$ was considered to indicate a statistically significant difference.

\section{Results}

LUT decreases UVA-induced cell death in HSFs. Previous studies have indicated that LUT has potential protective effects against exogenous oxidants in various cell types, however, these protective effects have not been investigated in HSFs exposed to UV irradiation $(6,9,12)$. To investigate the protective effects of LUT in human skin, the present study first investigated the resistance of cells to cell death induced by UVA irradiation (Fig. 1), following treatment of the cells with LUT at concentrations ranging between 0 and $40 \mu \mathrm{M}$.

The results of the MTT analysis showed the toxic effects of UVA towards the cells alone and following treatment with LUT. As shown in Fig. 2A and B, UVA irradiation induced HSF death in a dose-dependent manner. As shown in Fig. 2C, the HSFs exposed to UVA irradiation accompanied by incubation with LUT did not exhibit significant cell death, compared with the HSFs treated with UVA alone. These results indicated that LUT protected the HSFs from UVA-induced death.

LUT induces resistance to UVA-induced autophagy of HSFs. To observe the morphology of the HSFs following UVA irradiation and LUT incubation, a light microscope (Fig. 1) and fluorescence microscope were used to visualize the cells.

Autophagy, also termed non-apoptotic programmed cell death (type II programmed cell death), involves a series of biochemical steps, through which eukaryotic cell death is induced through self degradation of their own cytoplasm and organelles (17). To determine whether LUT treatment decreases UVA-induced HSF autophagy, the cells were observed under a fluorescence microscope to detect AVO formation following staining with MDC. Punctuation of MDC-positive cells were observed when treated with $5 \mu \mathrm{M}$ LUT, compared with $40 \mu \mathrm{M}$ LUT, as shown in Fig. 3A. As shown in Fig. 3B, following treatment with the higher concentration of $40 \mu \mathrm{M}$ LUT, AVO formation decreased. The formation of AVOs decreased sharply following $24 \mathrm{~h}$ of treatment with the combination of LUT. To further demonstrate the induction of autophagy, electron microscopy was performed, which is the Gold Standard method for confirmation of autophagy. It was found that, in all treatments, incubation with higher concentrations of LUT exhibited fewer AVOs (Fig. 3B). Almost no cell death was observed in the untreated cells (Fig. 3C).

LUT impairs the production of ROS induced by UVA irradiation in HSFs. Cell redox status changes between the equilibration of ROS and GSH. In addition, MMP disruption and cell apoptosis are always associated with the generation of intracellular ROS and depletion of GSH $(18,19)$. Therefore, the present study examined the levels of ROS in HSFs treated with UVA irradiation, with and without incubation with LUT. The ROS levels were examined by DCFH-DA. The rapid generation of ROS, which was between 1.89- and 1.30-fold faster, compared with the control, was detected following UVA treatment, as shown in Fig. 4. Incubation with LUT decreased UVA-induced ROS production in the HSFs following treatment 


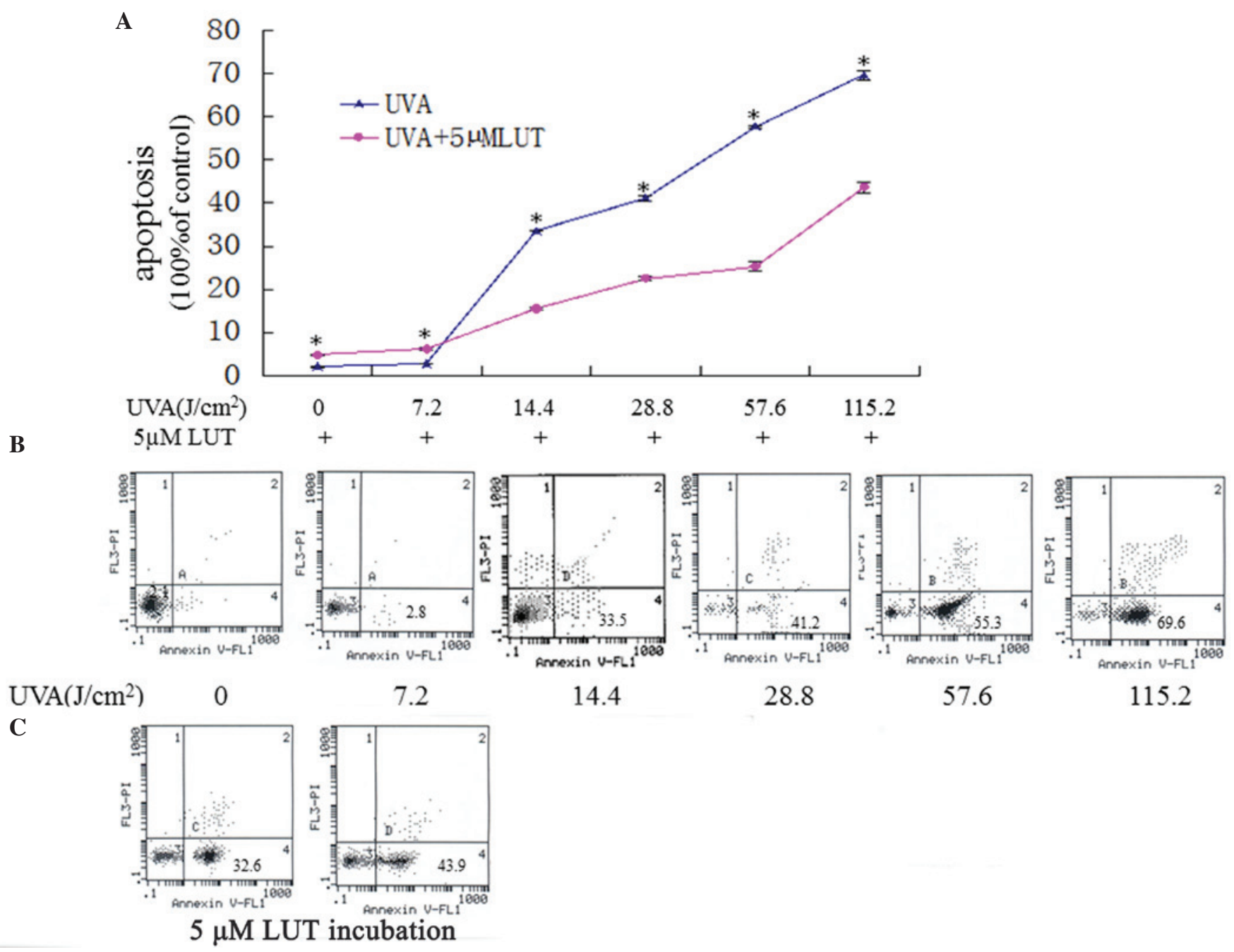

Figure 2. LUT decreases HSF apoptosis induced by UVA. The HSFs were assigned into two groups, and were irradiated with repetitive doses of UVA (0 J, $7.2,14.4,28.8,57.6$ and $115.2 \mathrm{~J} / \mathrm{cm}^{2}$, respectively). One group of HSFs was irradiated with UVA alone, the other group was irradiated with UVA and incubated with $5 \mu \mathrm{M}$ LUT. (A) 3-(4,5-dimethylthyl-thiazol-2-yl)-2,5-diphenyltetrazolium bromide analyses of the apoptosis in the two groups. Data are expressed as the mean \pm standard deviation ("P<0.05). (B and C) Annexin V-FITC/PI staining for the detection of apoptosis. The X-axis denotes Annexin V-FITC; the Y-axis denotes DNA content by PI. The apoptosis of HSFs were elevated gradually with the increasing doses of UVA while $5 \mu$ M LUT resisted HSFs apoptosis induced by UVA. The experiment was repeated three times, with representative results presented. HSFs, human skin fibroblasts; PI, propidium iodide; FITC, fluorescein isothiocyanate; UVA, ultraviolet A; LUT, luteolin.

with $40 \mu \mathrm{M}$ LUT, compared with the cells exposed to UVA alone. These results indicated that LUT assisted in impairing the UVA-induced increase in ROS levels in the HSFs.

Antioxidants protect HSFs from UVA cytotoxicity. The present study used two ROS scavengers, N-acetyl-cysteine (NAC), a well-known antioxidant and glutathione (GSH) precursor, and catalase (CAT; an $\mathrm{H}_{2} \mathrm{O}_{2}$-scavenging enzyme), to verify the linkage between ROS generation and cell toxicity in UVA-induced HSF cell death. Changes in MMP and cell toxicity were determined following exposure of the HSFs to UVA irradiation and incubation with either $1 \mathrm{mM}$ NAC or 2,000 U/ml CAT for $30 \mathrm{~min}$. As shown in Fig. 5A, the loss of MMP was inhibited by NAC and CAT, and the cells were protected from UVA cytotoxicity. Hoechst staining and a trypan blue exclusion assay indicated that NAC and CAT markedly reduced UVA-induced cell apoptosis. These results suggested that cell apoptosis was associated with ROS accumulation, followed by MMP disruption. As shown in Fig. 5B, LUT had the same effect as NAC and CAT, indicating that LUT exerted antioxidant effects against UVA irradiation in the HSFs.

Expression of HIF-1 $\alpha$ significantly decreases following UVA irradiation in HSFs incubated with LUT. Several cellular activities, including glycolysis, apoptosis, angiogenesis, metastasis and migration, are regulated by HIF-1, which is particularly sensitive to oxygen levels in the cell microenvironment (17,20-22).

Beclin 1 induces autophagy and inhibits tumorigenesis (22). To examine the expression of beclin 1 in the LUT-treated HSFs following UVA irradiation, western blot analysis was performed on the cell lysates. As expected, decreased expression levels of beclin 1 were detected following UVA exposure with LUT incubation (Fig. 6A), compared with the cells exposed to UVA alone. The present study also analyzed LC3, an autophagic marker, which is essential in the expansion of autophagosomes $(23,24)$. As shown in Fig. 6B, incubation with LUT in the UVA-irradiated HSFs led to a decrease in the conversion of LC3 from LC3-I, a soluble, cytoplasmic 
A

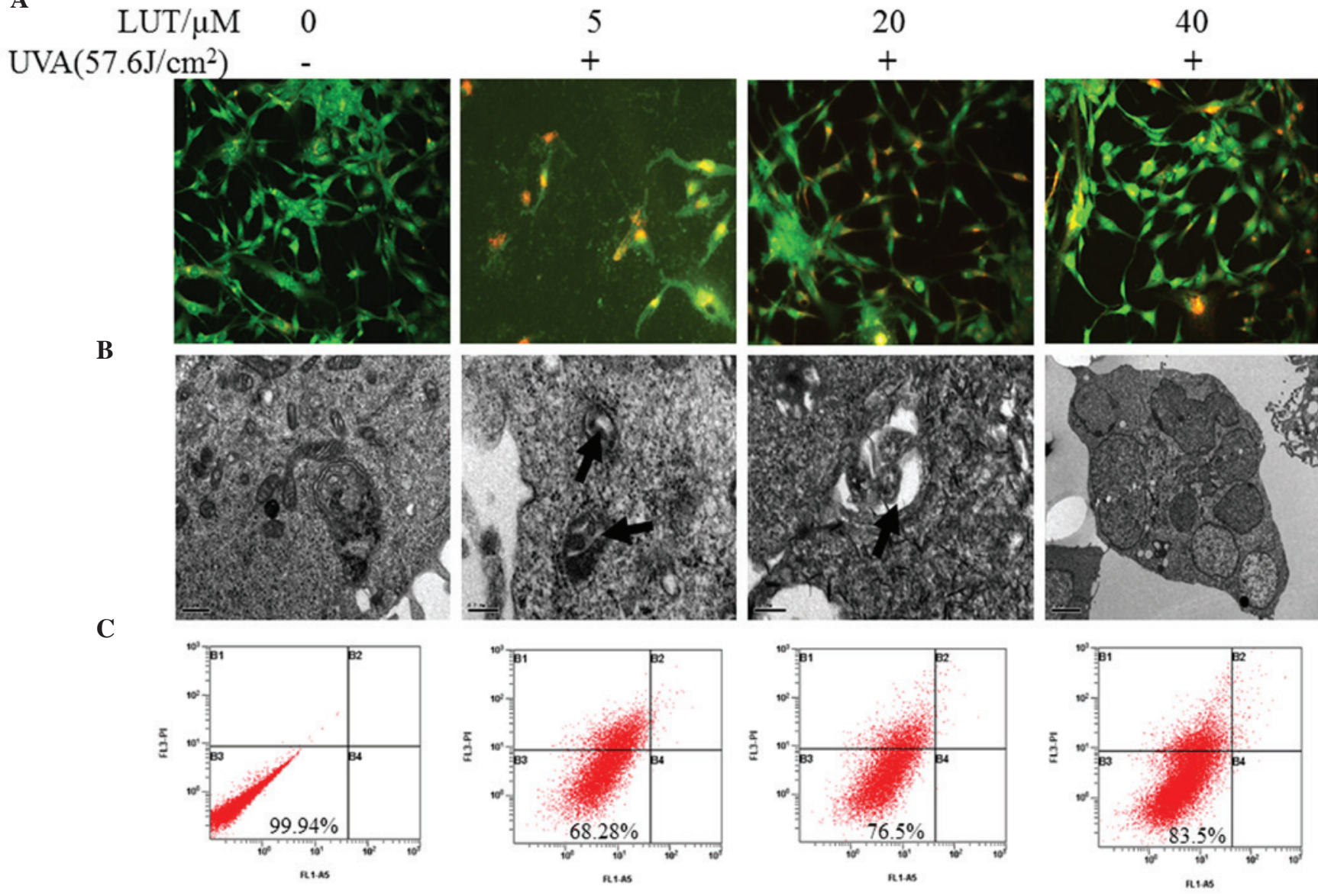

Figure 3. LUT decreases UVA-induced autophagy in HSFs. Four groups of HSFs were incubated with $0,5,20$ and $40 \mu \mathrm{M}$ LUT, respectively. These groups of HSFs were irradiated with $57.6 \mathrm{~J} / \mathrm{cm}^{2}$ UVA. (A) Following treatment, the cells were stained with MDC and observed using fluorescence microscopy to detect the presence of MDC puncta. (B) Electron micrographs of HSFs following treatment with LUT and irradiation with $57.6 \mathrm{~J} / \mathrm{cm}^{2} \mathrm{UVA}$. Phosphate-buffered saline was used in treatment as a bank control. Magnification, x200. (C) Annexin V-FITC and PI staining for apoptosis. The X-axis denotes Annexin V-FITC; the Y-axis denotes DNA content by PI. The experiment was repeated three times and the results are representative of the three independent experiments. HSFs, human skin fibroblasts; PI, propidium iodide; FITC, fluorescein isothiocyanate; UVA, ultraviolet A; LUT, luteolin.

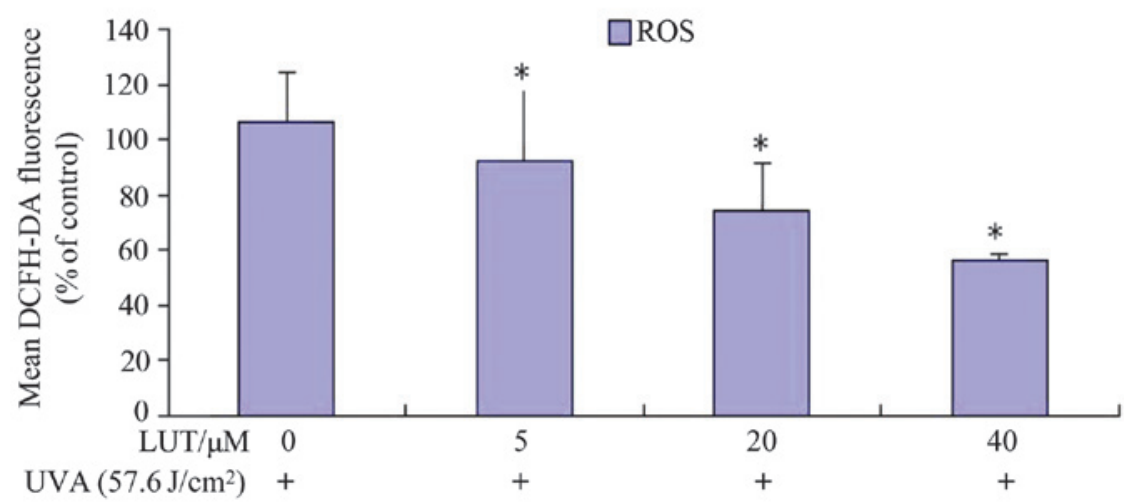

Figure 4. LUT decreases UVA-induced ROS in HSFs. The HSFs were incubated with different doses of LUT (0, 5, 20 and $40 \mu \mathrm{M}$ LUT, respectively) at a concentration of $1 \times 10^{6}$ cells $/ \mathrm{ml}$, and then exposed to $57.6 \mathrm{~J} / \mathrm{cm}^{2} \mathrm{UVA}$. Following three washes in PBS, the cells were incubated at $37^{\circ} \mathrm{C}$ for $30 \mathrm{~min}$ with $10 \mu \mathrm{M}$ DCFH-DA in PBS. The fluorescence was measured using flow cytometry (excitation wavelengths, $488 \mathrm{~nm}$; emission wavelengths, $525 \mathrm{~nm}$ ). The analysis was repeated three times, and the results are representative of the three. Each bar represents the mean \pm standard deviation of three experiments ("P<0.05, vs. dimethyl sulfoxide control). HSFs, human skin fibroblasts; UVA, ultraviolet A; LUT, luteolin; ROS, reactive oxygen species; DCFH-DA, dichlorodihydrofluorescein diacetate; PBS, phosphate-buffered saline.

form, to LC3-II, a membrane-bound, autophagosome-associated form. These results provided further evidence for the increased reduction of autophagy by LUT treatment in the UVA-irradiated HSFs.

\section{Discussion}

The antioxidant effect of LUT is due to several mechanisms. Firstly, the specific structure of LUT enables it to act as a 
A
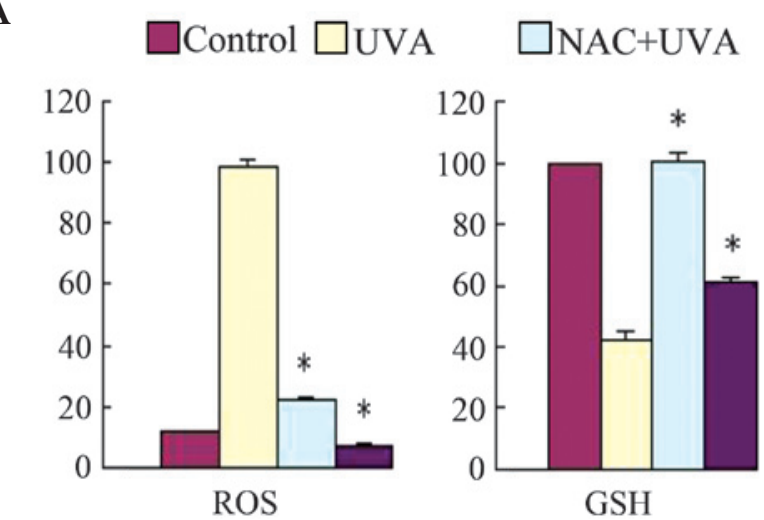

$\square$ CAT+UVA

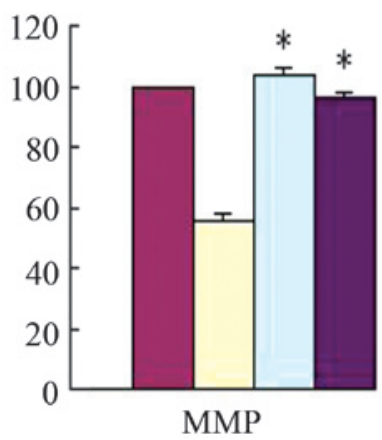

B

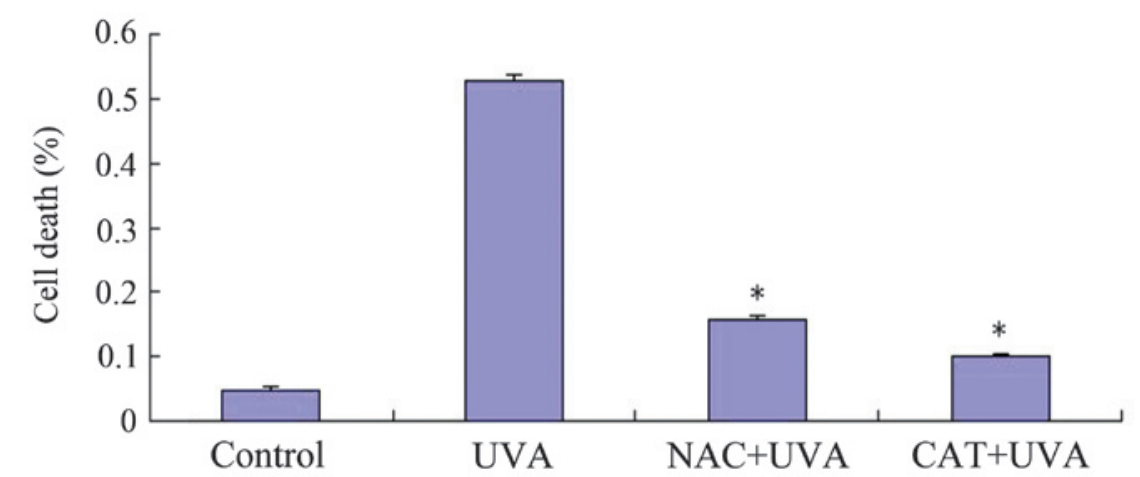

Figure 5. Effects of exogenous application of NAC and CAT on UVA-induced ROS generation, GSH depletion, MMP disruption and cell viability in HSFs (A) Changes in ROS, GSH and MMP were examined using flow cytometric analysis. (B) Cell death rate was determined using a trypan blue exclusion assay. Control, cells treated with solvent as control; UVA, cells treated with $57.6 \mathrm{~J} / \mathrm{cm}^{2} \mathrm{UVA}$; CAT+UVA, cells treated with $57.6 \mathrm{~J} / \mathrm{cm}^{2} \mathrm{UVA}$ following incubation with 2,000 U/ml CAT for $30 \mathrm{~min}$; NAC+UVA, cells treated with $57.6 \mathrm{~J} / \mathrm{cm}^{2}$ UVA following incubation with $1 \mathrm{mM}$ NAC for $30 \mathrm{~min}$. The data are expressed as the mean \pm standard deviation of three independent experiments; ${ }^{*} \mathrm{P}<0.05$, vs. cells treated with $57.6 \mathrm{~J} / \mathrm{cm}^{2} \mathrm{UVA}$ alone. HSFs, human skin fibroblasts; UVA, ultraviolet A; LUT, luteolin; ROS, reactive oxygen species; NAC, N-acetyl-cysteine; CAT, catalase; GSH, glutathione; MMP; mitochondrial membrane potential.

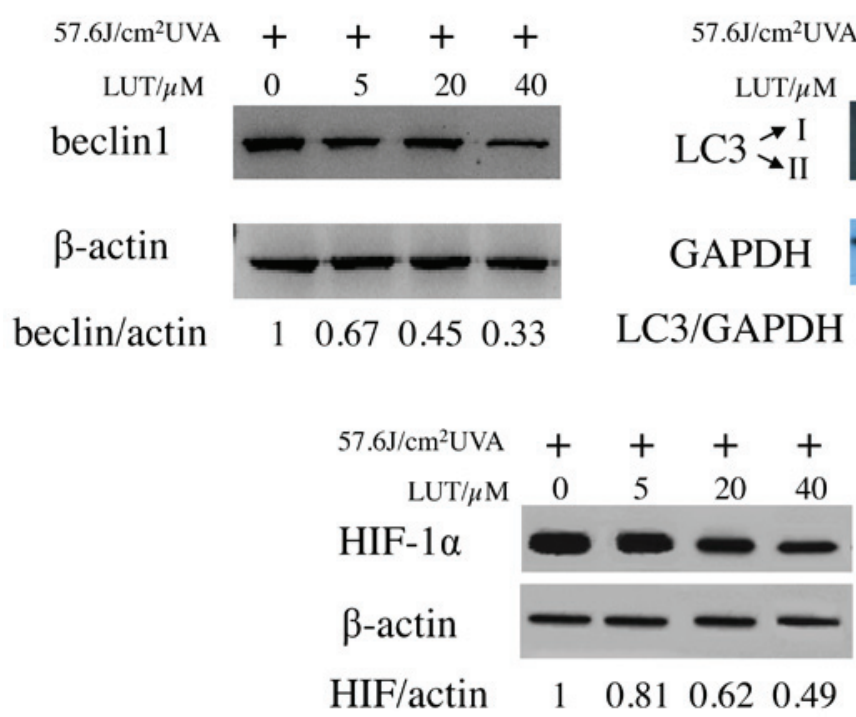

Figure 6. Effects of LUT on the expression levels of beclin 1, LC3 and HIF-1 $\alpha$ in UVA-irradiated HSFs. HSFs incubated with different concentrations of LUT $\left(0,5,20\right.$ and $40 \mu \mathrm{M}$, respectively) were treated with $57.6 \mathrm{~J} / \mathrm{cm}^{2} \mathrm{UVA}$. The cytosolic fractions were measured using western blot analysis to determine the expression levels of beclin1, LC3 and HIF-1 $\alpha$. The experiment was repeated three times and the blots shown are representative of the three independent experiments. HSFs, human skin fibroblasts; UVA, ultraviolet A; LUT, luteolin; HIF-1 $\alpha$, hypoxia-inducible factor-1 $\alpha$.

ROS scavenger (23). Secondly, ROS-generating oxidases, including xanthine oxidase activity, can be inhibited by LUT through suppressing $\mathrm{O}^{2} \bullet$-formation (25). It is already known that mitochondria are the primary site for ROS generation, however, whether LUT affects ROS generation in this manner in mammalian cells remains to be elucidated (26). Thirdly, intra- 
cellular antioxidant enzymes, including superoxide dismutase, glutathione-S-transferase, glutathione reductase and CAT may be protected or enhanced by LUT (27-29). Finally, LUT impairs the oxidation of several cellular components, possibly by inhibiting the activity of the corresponding enzymes.

Previous studies have indicated that various cell types show varying degrees of sensitivity to flavonoids (23-29). Verschooten et al (30) demonstrated that LUT decreases the damage induced by UVB-irradiation in normal human keratinocytes, whereas no photoprotective effects are observed in malignant keratinocytes.

The present study found that LUT exerted a protective effect on HSFs damaged by UVA. LUT was suggested to scavenge UVA-induced ROS in the HSFs. Of note, the UVA-irradiated HSFs endured substantial changes in autophagy when incubated with LUT, and these changes were due to decreases in the levels of ROS.

HIF-1 $\alpha$ is an important transcriptional factor induced by numerous oxidants. In the present study, HIF-1 $\alpha$ was downregulated in the LUT-treated UVA-irradiated HSFs. It was concluded that there are specific proteins downstream of HIF-1 $\alpha$, which induced autophagy. This indicated the association of HIF-1 $\alpha$ with autophagy. Once the expression of HIF-1 $\alpha$ decreased, autophagy declines. LUT, acting as a potent ROS scavenger, markedly impaired the production of HIF-1 $\alpha$ induced by UVA. These results indicated that LUT decreased the UVA-induced autophagy of the HSFs by scavenging ROS.

\section{Acknowledgements}

The present study was supported by the National Natural Science Foundation of China (grant no. 30972652), and the National Natural Science Foundation of Guangdong Province (grant no. 2013B021800053).

\section{References}

1. Seelinger G1, Merfort I, Wölfle U and Schempp CM Anti-carcinogenic effects of the flavonoid luteolin. Molecules 13: 2628-2651, 2008.

2. Rice-Evans CA, Miller NJ and Paganga G: Structure-antioxidant activity relationships of flavonoids and phenolic acids. Free Radic Biol Med 20: 933-956, 1996.

3. Mira L, Fernandez MT, Santos M, Rocha R, Florêncio MH and Jennings KR: Interactions of flavonoids with iron and copper ions: A mechanism for their antioxidant activity. Free Radic Res 36: 1199-1208, 2002

4. López-Lázaro M: Distribution and biological activities of the flavonoid luteolin. Mini Rev Med Chem 9: 31-59, 2009.

5. Masaki H, Atsumi T and Sakurai H: Detection of hydrogen peroxide and hydroxyl radicals in murine skin fibroblasts under UVB irradiation. Biochem Biophys Res Commun 206: 474-479, 1995.

6. Jurkiewicz BA and Buettner GR: EPR detection of free radicals in UV-irradiated skin: Mouse versus human. Photochem Photobiol 64: 918-922, 1996.

7. Barber LA, Spandau DF, Rathman SC, Murphy RC, Johnson CA, Kelley SW, Hurwitz SA and Travers JB: Expression of the platelet-activating receptor results in enhanced ultraviolet $\mathrm{B}$ radiation-induced apoptosis in a human epidermal cell line. J Biol Chem 273: 18891-18897, 1998.

8. Brenneisen P, Wenk J, Klotz LO, Wlaschek M, Briviba K, Krieg T, Sies H and Scharffetter-Kochanek K: Central role of ferrous/ferric iron in the ultraviolet $\mathrm{B}$ irradiation-mediated signaling pathway leading to increased interstitial collagenase (matrix-degrading metalloproteinase (MMP)-1) and stromelysin-1 (MMP-3) mRNA levels in cultured human dermal fibroblasts. J Biol Chem 273: 5279-5287, 1998.
9. Yasui $\mathrm{H}$ and Sakurai H: Chemiluminescent detection and imaging of reactive oxygen species in live mouse skin exposed to UVA. Biochem Biophys Res Commun 269: 131-136, 2000.

10. Kang S, Chung JH, Lee JH, Fisher GJ, Wan YS, Duell EA and Voorhees JJ: Topical N-acetyl cysteine and genistein prevent ultraviolet-light-induced signaling that leads to photoaging in human skin in vivo. J Invest Dermatol 120: 835-841, 2003.

11. Yamamoto Y: Role of active oxygen species and antioxidants in photoaging. J Dermatol Sci 27 (Suppl 1): S1-S4, 2001.

12. Hanson KM and Simon JD: Epidermal trans-urocanic acid and the UV-A-induced photoaging of the skin. Proc Natl Acad Sci USA 95: 10576-10578, 1998.

13. Shi Y, Wang $\mathrm{CH}$ and Gong XG: Apoptosis-inducing effects of two anthraquinones from Hedyotis diffusaWILLD. Biol Pharm Bull 31: 1075-1078, 2008.

14. Li J, Xu Z, Tan M, Su W and Gong X: 3-(4-(Benzo [d]thiazo 1-2-yl)-1-phenyl-1H-pyrazol-3-yl) phenyl acetate induced Hep $\mathrm{G} 2$ cell apoptosis through a ROS-mediated pathway. Chem Biol Interact 183: 183, 2010.

15. Biederbick A, Kern HF and Elsässer HP: Monodansylcadaverine (MDC) is a specific in vivo marker for autophagic vacuoles. Eur J Cell Biol 66: 3-14, 1995.

16. Gong K, Chen C, Zhan Y, Chen Y, Huang ZB and Li WH: Autophagy-related gene7 (Atg7) and reactive oxygen species/extracellular-signal-regulated kinase regulate tetrandrine-induced autophagy in human hepatocellular carcinoma. J Biol Chem 287: 35576-35888, 2012.

17. Maxwell PH, Dachs GU, Gleadle JM, Nicholls LG, Harris AL, Stratford IJ, Hankinson O, Pugh CW and Ratcliffe PJ: Hypoxia-inducible factor-1 modulates gene expression in solid tumors and influences both angiogenesis and tumor growth. Proc Natl Acad Sci USA 94: 8104-8109, 1997.

18. Xiong Y, Liu X, Lee CP, Chua BH and Ho YS: Attenuation of doxorubicin-induced contractile and mitochondrial dysfunction in mouse heart by cellular glutathione peroxidase. Free Radic Biol Med 41: 46-55, 2006.

19. Lopez E, Arce C, Oset-Gasque MJ, Cañadas S and González MP: Cadmium induces reactive oxygen species generation and lipid peroxidation in cortical neurons in culture. Free Radic Biol Med 40: 940-951, 2006.

20. Bardos JI and Ashcroft M: Negative and positive regulation of HIF-1: A complex network. Biochim Biophys Acta 1755: 107-120, 2005.

21. Harris AL: Hypoxia-a key regulatory factor in tumor growth. Nat Rev Cancer 2: 38-47, 2002.

22. Semenza GL: HIF-1, $\mathrm{O}^{2}$ and the 3PHDs: How animal cells signal hypoxia to the nucleus. Cell 107: 1-3, 2001.

23. Lien EJ, Ren S, Bui HH and Wang R: Quantitative structure-activity relationship analysis of phenolic antioxidants. Free Radic Biol Med 26: 285-294, 1999.

24. Shimoi K, Masuda S, Furugori M, Esaki S and Kinae N: Radioprotective effect of antioxidative flavonoids in gamma-ray irradiated mice. Carcinogenesis 15: 2669-2672, 1994.

25. Nagao A, Seki M and Kobayashi H: Inhibition of xanthine oxidase by flavonoids. Biosci Biotechnol Biochem 63: 1787-1790, 1999.

26. Sen N, Das BB, Ganguly A, Banerjee B, Sen T and Majumder HK: Leishmania donovani: Intracellular ATP level regulates apoptosis-like death in luteolin induced dyskinetoplastid cells. Exp Parasitol 114: 204-214, 2006.

27. Leung HW, Kuo CL, Yang WH, Lin CH and Lee HZ: Antioxidant enzymes activity involvement in luteolin-induced human lung squamous carcinoma CH27 cell apoptosis. Eur J Pharmacol 534: $12-18,2006$.

28. Manju V and Nalini N: Chemopreventive potential of luteolin during colon carcinogenesis induced by 1,2-dimethylhydrazine. Ital J Biochem 54: 268-275, 2005.

29. Harris GK, Qian Y, Leonard SS, Sbarra DC and Shi X: Luteolin and chrysin differentially Inhibit cyclooxygenase-2 expression and scavenge reactive oxygen species but similarly inhibit prostaglandin-E2 formation in RAW 264.7 cells. J Nutr 136: 1517-1521, 2006.

30. Verschooten L, Smaers K, Van Kelst S, Proby C, Maes D, Declercq L, Agostinis P and Garmyn M: The flavonoid luteolin increases the resistance of normal, but not malignant keratinocytes, against UVB-induced apoptosis. J Invest Dermatol 130: 2277-2285, 2010. 